\title{
Utilización de aplicaciones de teléfono móvil para elaborar un diario de actividad compartido durante el Máster en profesorado Using mobile phone applications to develop a shared activity journal during the Master's in teaching
}

\author{
José M. ${ }^{a}$ Falcó ${ }^{1}$, José Luis Huertas ${ }^{2}$ \\ chema.falco@unizar.es,jhuertas@unizar.es \\ ${ }^{1}$ Departamento de informática e Ingeniería de \\ Sistemas \\ ${ }^{2}$ Departamento de Ingeniería de Diseño y \\ Fabricación \\ Universidad de Zaragoza \\ Zaragoza, España
}

\begin{abstract}
Resumen- Las prácticas en los institutos de educación secundaria constituyen un elemento fundamental en la formación de los profesores. Durante este período es necesario el acompañamiento y la orientación por parte del tutor de la universidad. Este trabajo presenta una experiencia de innovación docente en la que se han utilizado una aplicación de mensajería móvil y una red social para realizar un diario de actividad compartido que ha permitido a los tutores realizar su labor de tutoría y seguimiento. Se efectuó en los cursos 2015-16 y 2016-17. La recogida de datos se hizo analizando los diálogos en ambas plataformas y recogiendo los testimonios de los alumnos en un cuestionario. Los resultados muestran que la experiencia ha sido un éxito porque, además de facilitar la presencia virtual del tutor durante las prácticas, desarrolló el sentimiento de grupo, propició la colaboración entre los alumnos y promovió la reflexión compartida sobre la práctica docente.
\end{abstract}

Palabras clave: Telegram, Prácticum, Máster en profesorado, Aplicaciones de mensajería instantánea

Abstract- Practices in secondary education institutions are a fundamental component in the training of teachers. During this period it is necessary the accompaniment and guidance by the tutor of the university. This article describes an innovative educational experience in which a mobile messaging application and a social network have been used to write a shared activity journal that has allowed tutors to carry out their tutorship and follow-up work. It took place in the 2015-16 and 2016-17 courses. Data collection was done by analyzing the dialogues on both platforms and student testimonies in a questionnaire. Results show that this experience was a success because, in addition to facilitating the virtual presence of the tutor during practices, it developed the group feeling, fostered collaboration among students and boost shared reflection on teaching practice.

Keywords: Telegram, Prácticum, Teacher training, Instant messaging applications

\section{INTRODUCCIÓN}

El período del prácticum que realizan en los institutos de educación secundaria quienes se están formando como docentes está cargado de tensiones. El soporte emocional tanto del tutor universitario como del grupo de clase es un elemento fundamental en esas circunstancias, pero al tener lugar en un contexto ajeno al espacio universitario "se evidencia ... que uno de los mayores problemas que se encuentra en la interrelación entre los distintos agentes se debe a las dificultades de comunicación entre las distintas partes" (Gastelaars \& Espasa, 2015, p. 5).
Raposo y Sarceda (2007) presentan a las herramientas de comunicación síncrona (chat y mensajería instantánea) y asíncrona (foro y correo electrónico) como aliadas para superar este problema en la realización de la tutoría y el seguimiento en la fase del prácticum. La tutorización online aparece repetidamente en los estudios analizados por Gastelaars y Espasa (2015) como un aspecto que potencia la comunicación y el apoyo durante estos períodos, que evidencia un contacto más continuado con el tutor de la universidad y que aumenta la sensación de cercanía entre los alumnos.

El teléfono móvil, que se ha convertido en el instrumento de comunicación más utilizado en el mundo por una amplia franja de edades, podría ser el soporte adecuado para llevar a cabo esta labor porque posibilita un contacto casi inmediato entre profesor y alumnos y entre alumnos y alumnos. Sin embargo, en el ámbito educativo, para los alumnos el móvil es un recurso lúdico y para la mayoría del profesorado un medio de distracción, por lo que su uso en actividades de aprendizaje todavía es muy limitado.

No obstante, algunos docentes lo están incorporando a su práctica. Bouhnik y Deshen (2014) han explorado los usos que se están dando a los grupos de clase de WhatsApp. Tres de estos usos - comunicarse con los alumnos, propiciar un clima de grupo y potenciar que los alumnos compartan y dialoguen entre sí - son necesarios en la situación del prácticum. En esta línea, Martín y Trigueros (2016) llevaron a cabo una investigación sobre la utilización de Messenger para realizar seminarios virtuales de tutorización del prácticum a distancia.

También parece que, aunque lentamente, se empieza a implantar entre el profesorado universitario el uso de las redes sociales, lo que facilita el contactar con un alumnado "que tiene su red de conexión social, que tiene su identidad en la red" (Duart, 2011).

Pero todavía son escasas las investigaciones en el uso de las aplicaciones de mensajería y redes sociales de manera continuada durante la realización de prácticas externas a la universidad. En esta comunicación presentamos los resultados de una experiencia de innovación docente llevada a cabo en el período del prácticum del Máster en profesorado que se ha centrado en integrar mediante la telefonía móvil una aplicación de mensajería y una red social como recurso para favorecer la comunicación y el apoyo entre los alumnos y el tutor de la universidad. Otro efecto que se persigue lograr es 
que los futuros docentes, al experimentar con la utilización didáctica de estas tecnologías, las acepten y las integren en su práctica educativa.

\section{Contexto}

Esta experiencia de innovación docente se ha realizado durante los cursos 2015-16 y 2016-17 en la asignatura Prácticum II de la especialidad de Tecnología e informática para ESO y Bachillerato del Máster en profesorado de la Universidad de Zaragoza. Este período de prácticas se desarrolla en institutos de Educación Secundaria y tiene una duración, descontando periodos de vacaciones, de cinco semanas.

Las prácticas que los estudiantes del Máster en profesorado realizan para completar su formación como futuros docentes se llevan a cabo en dos momentos: el Prácticum I, en el primer cuatrimestre, cuyo objetivo es la inmersión en la realidad del centro educativo y el Prácticum II, en el que deben diseñar, desarrollar y evaluar una unidad didáctica en una asignatura de su especialidad de un curso de ESO o Bachillerato, realizando una interacción entre la teoría aprendida en las aulas de la universidad y la práctica en las aulas de secundaria que facilite una reflexión sobre la práctica docente y ayude al futuro profesor a dar sentido y significación a la teoría.

Durante este tiempo trabajan y aprenden con la supervisión $\mathrm{y}$ orientación del tutor del instituto y con el acompañamiento y orientación del tutor de la universidad. En años anteriores, debido a la carga de trabajo de los estudiantes, ese acompañamiento se ha reducido a una visita por parte del tutor de la universidad al centro docente. Si dicho centro no se encontraba en la misma localidad o el horario no era compatible ni siquiera esta visita se efectuaba.

La evaluación de los aprendizajes adquiridos durante el prácticum la hacen, al $50 \%$, el tutor del instituto y el tutor de la universidad. Éste realiza su evaluación en base a tres documentos presentados por el estudiante: un diario en el que reflexiona sobre las diferentes actividades realizadas, un análisis comparativo de dos grupos de alumnos y la unidad didáctica que ha impartido.

La experiencia que se presenta tiene dos objetivos: 1) propiciar que el diario sea un elemento de reflexión personal sobre la práctica profesional y que sirva de vehículo para compartir la experiencia con el resto de los compañeros y 2) solventar las dificultades de acompañamiento, buscando que sea más continuo y que llegue a todos los estudiantes que están realizando el prácticum, superando las limitaciones que impiden la presencia física.

\section{DESCRIPCIÓN}

Las herramientas que han permitido al tutor de la universidad hacer un seguimiento continuado, han propiciado la reflexión individual $\mathrm{y}$ han facilitado que los alumnos compartan su experiencia durante la estancia en los institutos han sido Telegram y un foro web creado en la plataforma Moodle de la universidad, pero en este artículo solo vamos a analizar los resultados del uso de la primera.

Telegram es una aplicación de mensajería móvil gratuita que surgió en 2013. Tiene las características comunes a este tipo de aplicaciones, como son la de crear grupos y la de enviar e intercambiar información en distintos soportes (texto, voz, fotos, emoticonos, vídeos, música, enlaces URL y archivos) pero tiene además unas características que la hacen más adecuada para su utilización en ámbitos docentes que la más popular WhatsApp: a) Preserva la privacidad. Para comunicarse con otro usuario de Telegram basta con conocer su apodo en esta aplicación; no es necesario conocer su número de móvil. Esto permite que los docentes y los componentes del grupo que así lo deseen mantengan ese dato oculto. b) Permite el acceso vía móvil, vía aplicación de escritorio para los sistemas operativos Windows, Mac Os y Linux y vía web. c) Al igual que Twitter y otras redes sociales, permite mencionar a un usuario determinado en un chat de grupo. d) Permite unirse a un grupo mediante una invitación, lo que facilita la incorporación de los alumnos. Otra característica que hace de Telegram una aplicación adecuada para esta actividad es su gestión democrática. A diferencia de WhatsApp, en la que el creador de un grupo es su administrador de usuarios, en Telegram, salvo que el creador del grupo deshabilite esta opción, todos los miembros del mismo pueden dar de alta a nuevos miembros, lo que, nuevamente, facilitó la incorporación de los alumnos que no pudieron asistir a la reunión en la que los tutores de la universidad presentaron este formato para el diario.

En una reunión previa al inicio del Prácticum II se les dieron a los alumnos las pautas de realización del diario. Debían participar en el chat del grupo de la especialidad o en Twitter al menos una vez al día de lunes a viernes y hacer como mínimo una aportación semanal en un hilo del foro, bien abriendo un nuevo tema de debate, bien aportando su reflexión en un hilo abierto por otro compañero. En ambos medios las aportaciones tenían que guardar relación con su experiencia en el instituto o con un tema de interés para la práctica docente. En esa reunión se acordó el nombre del grupo, que coincidía con la etiqueta (o \#hastag) a utilizar en Twitter y se les facilitó el nombre de usuario de los tutores. Posteriormente, una vez creado el grupo, se les proporcionó el enlace para que se unieran.

Uno de los tutores de la universidad saludaba al iniciar la mañana a todo el grupo en Telegram y publicaba el mismo saludo en Twitter con la etiqueta acordada. El saludo, cuando era conveniente, hacía referencia a alguno de los comentarios del día anterior. El resto de los días planteaba retos que afrontar como docentes en la nueva jornada. Además, los tutores intervenían al menos una vez a lo largo del día, contestando a los comentarios de los alumnos, proponiendo alternativas a las cuestiones planteadas o transmitiendo ánimos $\mathrm{y}$ felicitaciones.

Aunque a los alumnos se les propuso realizar el diario en Telegram o en Twitter utilizando la etiqueta acordada, solo una alumna extranjera, sin número de móvil español, utilizó la red social de los 140 caracteres en el curso 2015-16. El resto de los alumnos de ese curso y todos los del 2016-17 han preferido un grupo privado.

La recogida de información, su análisis e interpretación de los resultados, se ha hecho a partir del contenido de las conversaciones extraídas de los chat de los dos grupos, uno cada curso, de la valoración cualitativa que mediante un cuestionario anónimo contestaron los alumnos sobre qué les había aportado la utilización de Telegram a su experiencia durante el prácticum y de los datos cuantitativos del número de participaciones. El cuestionario se les pasó online una vez finalizado el periodo de estancia en los institutos. 


\section{Resultados}

La muestra está formada por los 22 alumnos que realizaron el prácticum en el curso 2015-16 y los 21 que lo han realizado en el curso 2016-17. El cuestionario fue contestado por un $82 \%$ de los alumnos de $2015-16$ y por un $76 \%$ en $2016-17$ (18 y 16 alumnos respectivamente)

\section{A. Participación}

La participación en la actividad ha sido mayoritaria en ambos cursos, tal como queda reflejado en la Tabla 1. Solo un alumno $(6,25 \%)$ dejó de aportar su comentario o reflexión en más de cuatro ocasiones en el curso 2016-17.

Tabla 1: Datos de participación

\begin{tabular}{rrrr}
\hline Participación & Diaria & fallo 3 & fallo 4 a 10 \\
\hline 2016 & $94,44 \%$ & $5,56 \%$ & $0,00 \%$ \\
2017 & $81,25 \%$ & $12,50 \%$ & $6,25 \%$ \\
\hline
\end{tabular}

Como ya ha quedado indicado, el canal casi unánime de participación ha sido Telegram. Las razones para no utilizar Twitter son fundamentalmente tres. En primer lugar la comodidad (un 50\% de los participantes manifestaron esta razón), por ser una herramienta similar a WhatsApp, porque "no está limitado a 140 caracteres" o "El número de letras no está contabilizado", porque "la interfaz para las respuestas a cada comentario, es decir, más adecuado como chat para interactuar entre nosotros" y porque "como puede instalarse en el ordenador me parece muy cómoda para escribir las experiencias propias". En segundo lugar porque el feed-back es más inmediato, ya que o bien "no recibía feed-back en Twitter" o bien "Twitter no genera ese feedback inmediato" y en cambio en Telegram "parece un buen sistema para intercambiar opiniones entre los compañeros casi inmediatamente". En tercer lugar por la privacidad ya que Telegram "no es público" mientras que en Twitter "los mensajes son públicos, es decir los ve gente ajena al máster".

\section{B. Valoración de las respuestas directas}

Para conocer la valoración de los alumnos se les preguntó por su preferencia en cuanto al soporte del diario que debían realizar durante el Prácticum II, por su nivel de acuerdo o desacuerdo con las afirmaciones recogidas en la Tabla 2, pidiéndoles que resaltaran el aspecto que más valoraban de la utilización de Telegram, y por su valoración del papel de los tutores de la universidad.

La opción mayoritaria para el soporte ha cambiado de un año a otro (Fig. 1). Mientras que en el curso 2015-16 la preferencia fue haber utilizado exclusivamente Telegram en el curso 2016-17 ha sido haber utilizado ambas herramientas: Telegram y foro. Solo un alumno manifestó que optaría por el soporte tradicional, un documento al finalizar el prácticum, porque le "estresa el tener que estar pendiente de 3 cuentas de correo en el trabajo, más otro grupo de Telegram, más otros 3 de whatsapp." El resto, un $97 \%$ de los participantes, han optado por un formato abierto y compartido - "Me ha gustado más que hacer un simple diario para el prácticum" - porque valoran la participación de todos en su elaboración frente a un documento privado que solo lea el profesor: "Me parece bien las dos herramientas utilizadas, siempre los compañeros pueden aportar su punto de vista a un problema que tengas y crecer con los comentarios en lugar de hacer un diario para ti que se lo lee el profesor y ya está" o "El ser tan participativo y en común con el resto de compañer@s es lo que más me ha gustado, porque creo que es mucho más adecuado que simplemente plasmarlo en un documento escrito y privado que quede sólo entre el tutor y el alumno".
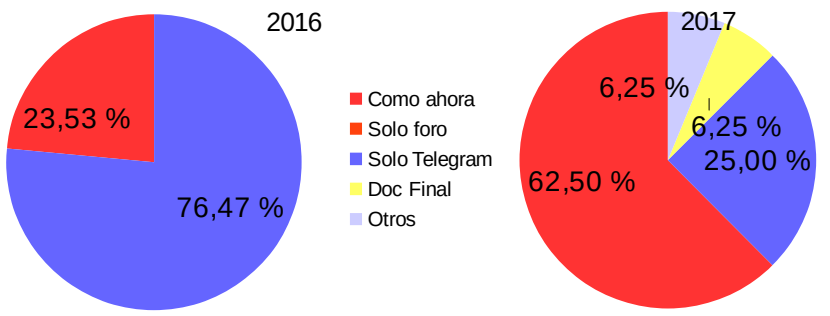

Figura 1: Preferencia de soporte para el diario

La utilización de Telegram como herramienta para compartir, sentir la pertenencia a un grupo y reflexionar sobre la práctica diaria ha sido bien valorada. Se les preguntó por su total desacuerdo (puntuado con 0) o total acuerdo (puntuado con 5) con las afirmaciones recogidas en la Tabla 2. En las dos ediciones de la experiencia lo más valorado ha sido compartir la experiencia (4 en 2016 y 4,13 en 2017). También lo ha sido "Sentirse grupo" que ha tenido una valoración cercana al 4 (3,94 en 2016 y 4 en 2017). El conjunto de las valoraciones está recogido en la Figura 2.

Tabla 2: Participar en Telegram te ha servido para
A Sentirme grupo con el resto de mis compañeros de máster
B Reflexionar sobre mi práctica diaria
C Recibir el apoyo y/ o las sugerencias de los compañe- ros
D Compartir mi experiencia
E Pararme a pensar unos minutos sobre lo que he hecho en clase cada día
F Comentar y conocer cómo les "ha ido el día" a mis compañeros
G Sentir la 'compañía' del tutor de la universidad durante el prácticum

Nada. Nada. Lo considero una pérdida de tiempo

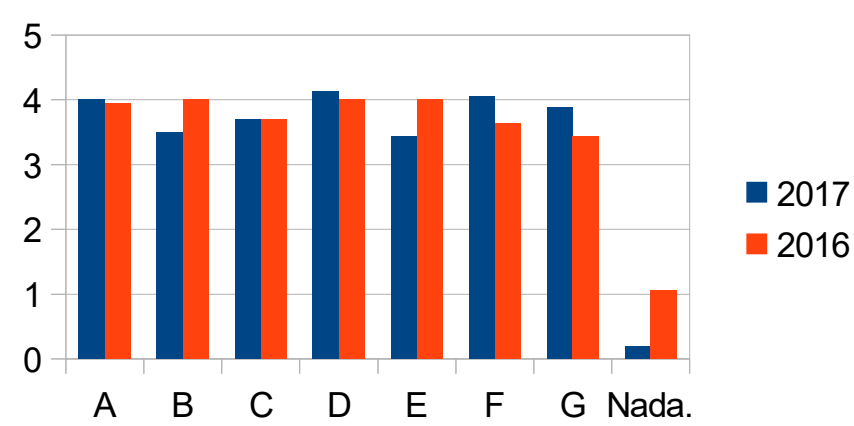

Figura 2: Participar en Telegram te ha servido para...

Individualmente lo más valorado ha sido compartir:

"Conocer el funcionamiento de otros centros", "leer las 
distintas experiencias que ha tenido cada alumno", "Compartir opiniones, consejos, sugerencias y el apoyo entre compañeros-tutor", "no perdimos la relación durante estos meses y nos dimos ideas unos a otros (el kahoot que se fue contagiando)", "Que puedes ayudar a tus compañeros" o "ver si el resto de compañeros pueden tener los mismos problemas que tú, y además permite que tus compañeros, o tutor, puedan opinar, aconsejar, o recomendar lo que ellos harían ante determinadas situaciones" son algunas de las opiniones que han manifestado. También han valorado "La espontaneidad" y "la frescura", "el feedback casi inmediato", "1a inmediatez y comodidad" y el aspecto práctico: "Es pragmático y te obliga ha realizar el diario de forma continuada" o "Aunque habia días que se hacía pesado luego se agradece no tener que escribir un diario".

También se les pidió su valoración de 0 a 5 sobre cómo les habían ayudado las aportaciones propias, de sus compañeros y del profesor. En 2016 la aportación más valorada fue la propia $(3,44)$ y la menos valorada la del profesor $(3,31)$ mientras que en 2017 la del profesor fue la más valorada $(3,94)$ y la que menos la propia $(3,38)$. El resumen está recogido en la Figura 3.

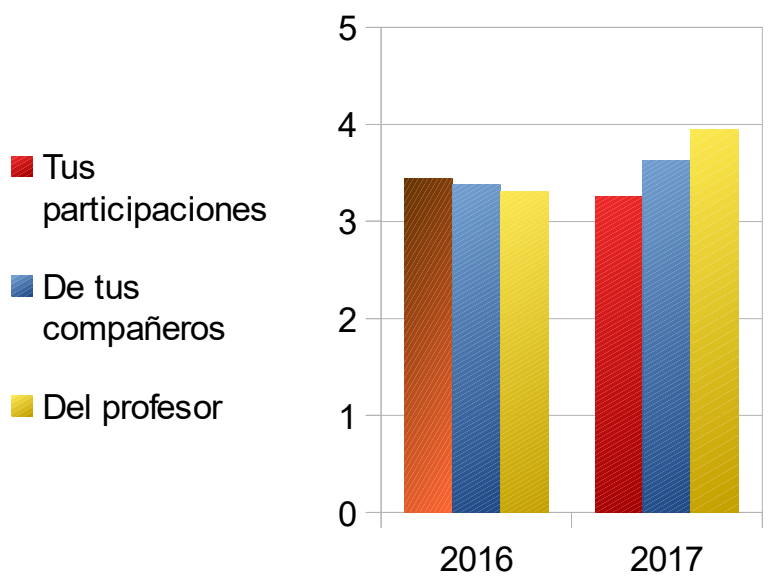

Figura 3: ¿Cómo te han ayudado las participaciones?

Los estudiantes han considerado la participación de los tutores en el chat del grupo un componente esencial. Si no hubieran participado habrían considerado que "no esta interesado", que "no se involucra en las actividades que propone" y que "no tiene interés por nosotros" además de echar "en falta la opinión de un docente con años de experiencia". En cambio "al haber recibido asistencia por parte de mi tutor, he visto reconocido mi esfuerzo y ha ayudado a entender ciertas cosas que pasaban durante el practicum". De hecho, dos de los alumnos de 2015-16 se quejaron de que su "tutor de la universidad no ha participado. Me hubiera gustado que lo hiciese para que viera cómo me estaba yendo la experiencia".

También han valorado positivamente el saludo al iniciar cada jornada porque "era motivador leerlo antes de comenzar las clases", "resultaba motivante y divertido en alguna ocasion, je je", "me alegraba las mañanas al sentir que uno de nuestros profesores pensaba en como nos iría", "era bueno para reflexionar" y "una buena píldora para empezar el día y focalizar en algo concreto". No obstante, hay quien los ha

1 Las intervenciones de los alumnos se han transcrito de forma literal, por lo que puede incluir abreviaturas o errores ortográficos o gramaticales. considerado "muy optimista. Casi utópico" o con "demasiado sabor a arenga".

\section{Análisis de los chat}

Tras analizar las conversaciones de ambas ediciones se ha refrendado la opinión de los alumnos de que la utilización de Telegram ha servido para crear grupo, para compartir las experiencias prestarse ayuda y colaboración, para reflexionar sobre la práctica docente y para compartir distintos materiales.

Han creado grupo. Los alumnos se animaban (Fig 4), se felicitaban por los logros y compartían emociones ("Bueno yo hoy estoy pletórico contentísimo de todo", "Yo hoy no sé cómo contarlo ... Ha sido como una montaña rusa", "Día de muchas emociones! [emoticono sonriendo]" o "Día bastante aburridete, de observador").

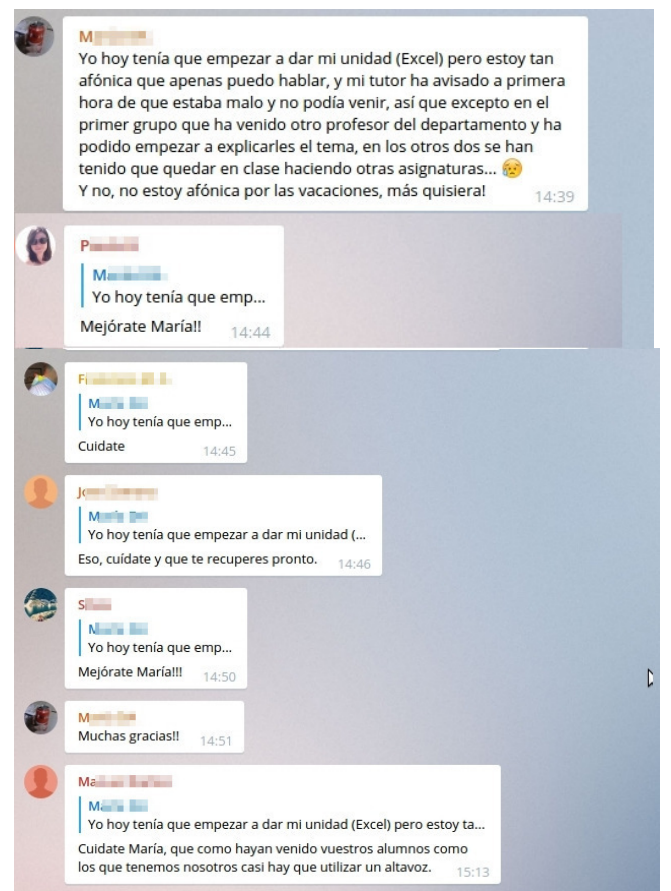

Figura 4: Apoyo emocional

Además de compartir experiencias se han ayudado y hecho sugerencias:

Alumna 1: A mi me encantaría poder usarlo [Kahoot]!! Tiene muy buena pinta por lo que comentais!! Pero está prohibidisimo el móvil en clase...

Alumna 2: Axxxx yo lo uso con los minis, no se si en tu Instituto teneis

En otra conversación:

Alumno 1: Se me plantea la siguiente duda: $\mathrm{Si}$ recurrimos a la gamificación/ludificación en secundaria para mantener la motivación. Hasta qué edad?

Alumna 3: Yo decirte que a mí me está funcionando bastante bien. Hoy una alumna le decía a otra ...

Han reflexionado sobre la práctica docente y han compartido su reflexión:

Alumno 2: Yo gracias a las jornadas interdisciplinares he podido ver como responden a 
una misma actividad alumnos de niveles distintos y observar las diferencias en las reflexiones y los comentarios según su edad

Alumno 3: Vaya diferencia entre una clase a las 9:00 y otra a las 14:00!! Como se nota que se van alterando a medida que transcurre la mañana.

Alumna 4: En mi evaluacion, una profe, que decía que casi todos sus alumnos iban a suspender en Junio. Pues ya sabéis según el Pigmallion...

Alumno 4: creo q deberían darle una vuelta a determinados talleres para $\mathrm{q}$ sean más útiles para ellos y motivantes

Cuando los tutores de la universidad consideraban que algunas intervenciones requerían un análisis más sosegado (efecto Pigmalión, libro de texto o libro electrónico, necesidad de conocer la situación personal de cada alumno, ... ) animaban a quien la había iniciado a que abriera un hilo en el foro para propiciar que todos los compañeros pudieran aportar su reflexión de una manera más pausada.

Los alumnos han utilizado Telegram para compartir distintos materiales como noticias relacionadas con la profesión docente ("El 'bullying' también es responsabilidad de los padres " o "Alerta por el juego suicida de la Ballena Azul"), vídeos o enlaces a documentales y webs que consideran de interés ("En la web de mi instituto han puesto acertijos matemáticos.”)

Las aportaciones hacían referencia a acciones o situaciones ya ocurridas y se hacían en momentos de descanso o una vez finalizado el horario de clases (ver hora de emisión de los mensajes en la Figura 4). La realización del diario con el móvil no ha interferido durante su actuación en las aulas, sino que la ha reforzado al recibir consejo y apoyo de compañeros $\mathrm{y}$ tutores.

Aunque según Doering y otros (2008) los profesores son reacios a utilizar aplicaciones de mensajería móvil porque temen que se llenen de mensajes irrelevantes y sin sentido esto no ha ocurrido en ninguna de las dos ediciones. Todas las participaciones, salvo quizás una felicitación a la llegada de la primavera o las despedidas al iniciar las vacaciones de Semana Santa, han sido relevantes para el contexto de las prácticas docentes. La utilización del grupo de Telegram se ha limitado a aquello para lo que se creó y no ha producido una sobrecarga de información.

\section{CONCLuSiOnes}

A partir de los resultados expuestos y analizados en este artículo se puede concluir que los alumnos prefieren un formato abierto y compartido para la realización del diario que deben elaborar durante el prácticum y que optan por la aplicación de mensajería móvil Telegram frente a la red social Twitter para la realización de un diario compartido. El uso de esta aplicación no solo favorece la labor de tutoría y seguimiento durante el prácticum, propiciando que los alumnos sientan cercana la presencia virtual de su tutor, sino que además sirve para potenciar el grupo y para desarrollar prácticas colaborativas.

Otros aspectos positivos son que los alumnos han reflexionado de manera conjunta sobre aspectos propios de la práctica docente y que se han comprometido con la actividad, implicándose al comunicar de manera continuada cómo estaban viviendo la experiencia y compartiendo distintos materiales.

En resumen, Telegram proporciona al tutor de universidad información "a tiempo real" de los logros y problemas de los estudiantes que están realizando las prácticas, proporciona a los estudiantes un canal de comunicación por el que compartir experiencias y proporcionarse ayuda y acompañamiento emocional y permite al tutor hacerse presente en el día a día de los estudiantes. Por ello consideramos que es una herramienta adecuada para hacer el seguimiento y acompañamiento de las prácticas externas que los estudiantes de cualquier titulación realizan como parte de su formación.

Hay que resaltar que para transferir esta actividad a otros grados que incluyan estos períodos de prácticas externas a la universidad o al Prácticum de esta titulación en otras universidades se requiere de un contexto que permita la dedicación diaria de los tutores, de manera breve pero continuada. Si el tutor no puede hacerse presente con respuestas, opiniones, comentarios y sugerencias en el chat del grupo la actividad carece de sentido para los estudiantes y él pierde la oportunidad de la intervención casi inmediata que le proporciona la aplicación.

Además de ser la opción preferida por los alumnos, la gratuidad de Telegram, su formato multiplataforma y su facilidad de uso hacen que sea una actividad sostenible.

\section{REFERENCIAS}

Bouhnik, D., \& Deshen, M. (2014). WhatsApp goes to school: Mobile instant messaging between teachers and students. Journal of Information Technology Education: Research, 13, 217-231. Recuperado el 8 jun 2017 de http://www.jite.org/documents/Vol13/JITEv13Research P217-231Bouhnik0601.pdf

Doering, A., Lewis, C., Veletsianos, G., \& Nichols-Besel, K. (2008). Preservice teachers' perceptions of instant messaging in two educational contexts. Journal of Computing in Teacher Education, 25(1), 5-12.

Duart, J. (2011). La red en los procesos de enseñanza de la Universidad. Comunicar, 37(19), 10-13.

Gastelaars, T., \& Espasa, A. (2015). Uso del Campus Virtual en el proceso del practicum en un contexto universitario de modalidad presencial. Revista de Educación a Distancia, (2DU). Recuperado el 6 de junio de 2017 de https://www.um.es/ead/reddusc/2/gastelaars.pdf

Martín, J. M., \& Trigueros, C. (2016). Mensajería instantánea y construcción compartida de significados: una experiencia de aprendizaje colaborativo en el Practicum de Maestro de Educación Primaria. Revista de Educación a Distancia, (51). Recuperado el 20 de mayo de 2017 de http://www.um.es/ead/red/51/martin_trigueros.pdf

Raposo, M. \& Sarceda, M.C. (2007). La integración de las TIC en la supervisión del Practicum. En Raposo, M y otros (Coords). Actas del IX Symposio Internacional sobre el Practicum (pp. 1091-1101). Poio (Pontevedra): Imprenta Universitaria. 